\title{
Numerical simulations for stochastic meme epidemic model
}

\author{
Ali Raza', Muhammad Rafiq ${ }^{2}$, Dumitru Baleanu ${ }^{3,4,5^{*}}$ and Muhammad Shoaib Arif ${ }^{1}$
}

\section{"Correspondence:}

dumitru@cankaya.edu.tr

${ }^{3}$ Department of Mathematics, Cankaya University, Ankara, Turkey

${ }^{4}$ Department of Medical Research, China Medical University Hospital,

China Medical University, Taichung, Taiwan

Full list of author information is available at the end of the article

\section{Springer}

\begin{abstract}
The primary purpose of this study is to perform the comparison of deterministic and stochastic modeling. The effect of threshold number is also observed in this model. For numerical simulations, we have developed some stochastic explicit approaches, but they are dependent on time step size. The implicitly driven explicit approach has been developed for a stochastic meme model. The proposed approach is always independent of time step size. Also, we have presented theorems in support of convergence of the proposed approach for the stochastic meme model.
\end{abstract}

Keywords: Meme model; Stochastic approaches; Convergence analysis

\section{Literature survey}

Dawkins elaborated the meme for the first time as "a unit of cultural transmission, or a unit of imitation" [1]. Nowadays, due to calm tech communication between the individuals, transfer of memes occurs more swiftly than ever before. Examples of the meme are a way of living like dining, wearing, and thinking. Propagation of meme and spread of rumor are quite similar to each other and act like a virus that can blow out through the population due to listening, viewing, reading, or some other way. An epidemiological model is primarily utilized by researchers to describe the dynamics of the social system. Mainly this model is used to study the changing aspects of rumors spread and transfer of opinions. This model is based on the fact that human diseases and public behavior are a result of interaction between the entities of society. Among the earliest researchers, Daley and Kendall were the prominent ones who proposed that a rumor spread model and an epidemic model are alike in [2]. Cane presented that various forms of a deterministic model for the spread of epidemic or rumor are the same in [3]. At the start of this century, numerous scientists have studied dynamics of rumors through multiple forms of the epidemiological model. Kawachi et al. [4] suggested the deterministic model for constant and variable gossip in an age-independent population; however, later, he found out the effect of various contact interactions in a rumor transmission model. Bettencourt [5] and Wang and Wood [6] both have applied epidemiological model, the former for the spread of ideas and the latter for the viral meme propagation. Two separate scientists Piqueira and Haung studied the spread of rumors. Piqueira studied the equilibrium of rumor spread model according

(c) The Author(s) 2020. This article is licensed under a Creative Commons Attribution 4.0 International License, which permits use sharing, adaptation, distribution and reproduction in any medium or format, as long as you give appropriate credit to the original author(s) and the source, provide a link to the Creative Commons licence, and indicate if changes were made. The images or other third party material in this article are included in the article's Creative Commons licence, unless indicated otherwise in a credit line to the material. If material is not included in the article's Creative Commons licence and your intended use is not permitted by statutory regulation or exceeds the permitted use, you will need to obtain permission directly from the copyright holder. To view a copy of this licence, visit http://creativecommons.org/licenses/by/4.0/. 
to propagation parameters and initial condition [7]. Haung investigated its range by applying denial and skepticism [8]. Zhao et al. did another addition in rumor spreading. He proposed a rumor spread model considering the forgetting mechanism of spread in the social network $[9,10]$. Huo et al. studied the dynamics of rumor transmission model with incubation mechanism [11].

On the other hand, Thompson explored the dynamics of rumor spread in a chat room [12]. Al-Amoudi et al. quantitatively analyzed a meme propagation model of a constant meme [13]. Rumor is essential to forming public communication, and its transmission has important influence on the population or society. Research related to the spread of memes has its significance. It provides a public drive to generate specific ideas, to change the perception of something, and to persuade others. Rumor may contain secret information regarding renowned persons and news that concerns critical social issues. It can affect human beliefs and reshape public opinion by changing the market level of some product. Its distribution plays a substantial part in various human activities. Different scientists define it differently; for example, Hayakawa defines it as a "Type of societal interact in which a false piece of information spread in population or community in a short period through the public link". Another scientist Shibutani redefines rumor as a shared problem solving, in which people trapped in a complicated situation try to find out meaningful results by collectively utilizing all of their cognitive powers. Rumor transmission is "social contagion processes".

In this paper, we use models like epidemiology for rumor spread and cessation. Track of study to the spread of rumor is highly reliable on the epidemiological model. This is because of revolutionary contributions to the modeling. It involves the dispersion of ailment and the elimination of affected persons. The rumor transmission model divides the individuals into three classes referred to as the susceptible, the infected, and the recovered. These classes represent the epidemiological status of subjects; therefore, these models can calculate average disease development by calculating the average of the population who are infested, who are possible to suffer, and those who have improved from disorder over some time. The term "rumor" refers to a fickler piece of information that can move from person to person or a vague piece of knowledge, and it takes time to separate what is wrong and right.

Despite its definition, the important thing is to possibly tell if an individual has adopted, remembered, and spread it. Daley and Thompson were the earlier scientists who introduced the conventional representations for the rumor transmission, and then many scholars have followed the model extensively in the past for their numerical studies. This model has divided the individuals into three classes. Ignorants (who are not aware of the rumor), spreaders (who spread it through social interaction), and stiflers (who have vague information but stop spreading it after talking to a subject who is already aware of that, and they have pairwise interaction). The findings of model revealed that when the spreader contacts the ignorant, this may change him to spreader, while spreader contact will convert both spreaders to stiflers. Spreader-stifler interaction will stifle the spreader. Another work of Kawachi, along with his co-workers on the plasticity of the spreader-ignorant-stifler model, revealed that spreader-to-ignorant and stifler-to-spreader transitions are possible. The spreading process of rumor with many different ideas was studied by Bettencourt et al., whereas Huang established two models to accommodate sceptics and found out 
the news dispersal procedure with denial and skepticism. In the Maki-Thompson model, spreader-spreader contact will cause a rumor to spread but stifler will be the initiating one.

There are lots of studies proposed by various scientists who studied a very complex model for the spread of false information based on several classical models. These studies were connected with scale-free networks, like uncorrelated and assortative degree correlation, and also were based on networks that were internet-mediated, such as "virtual" communities and email networks. The main conclusion of these representations is that they deserted the topological characteristic of social networking, and roughly these were not appropriate for extensive dispersal procedure. Moreno et al. have done the study of stochastic model based on scale-free networks.

He claimed that the standardization of systems profoundly influences the dynamic mechanism of rumor spreading. Sudbury related the active process of rumor transmission on media, and he claimed that the versatility of the ways involved in the spread of news matched that of the SIR model. Isham et al. revealed the final size distribution on the global network. Zanette and Buzna et al. did work on the rumor circulating critical value, and the final percentage of rumor heard in a population was done by Liu et al.

Various mathematical models during the past few centuries have developed for the study of the transmission of rumor within the population. There is a fundamental difference between the spread of rumor and disease beyond qualitative parallels. Unlike a disease, the spread of rumor is an intentional act committed by the spreader and the receiver. Rumors always require authenticity and take time to identify, for example, news that needs confirmation is the core element connected with rumor. One should not ever believe rumor without differentiating between true and false and should not transmit it without knowing the truthfulness of news. Social circle around an individual is an essential source of information along with some readable resources from which an individual can find information. People do not believe in network information immediately because of its weak credibility, but they believe in it when they hear it from their friends or relatives. Mostly rumors come from the network and spread from person to person, and sometimes a person does not spread it and keeps it into one's own heart until he or she becomes a transmitter or stifler in reality. Interest in spreading of information is an essential factor in its transmission, so the classic rumor transmission model required to be improved and perfected. Mathematical modeling has become very advanced to understand the epidemic diseases thoroughly. Formation of models and simulation allows us to analyze the sensitivity and make a comparison of conclusive opinions originating out of examples. This allows anticipating mediators, environmental elements influencing, and public health. As a result, higher authorities (policy makers) can scientifically implement or suggest safety measures. Lots of studies have presented various models of meme transmission dynamics [11-13].

However, stochastic explicit approaches do not maintain the dynamical properties in the stochastic meme model. So, the following question arises: Do we conserve all the dynamic features in a stochastic numerical approach? Our main purpose of this paper is to introduce a stochastic approach that does not violate the dynamical properties, namely as stochastic nonstandard finite difference scheme (SNSFD) presented in [14-16].

This paper has adopted the following strategy:

In Sect. 2, we present a deterministic meme model and its equilibria. We look at the construction of the stochastic meme model in Sect. 3. We present the different stochastic approaches and their contrast in Sect. 4. In Sect. 5, we give conclusion and directions. 
Figure 1 Flow map of meme model

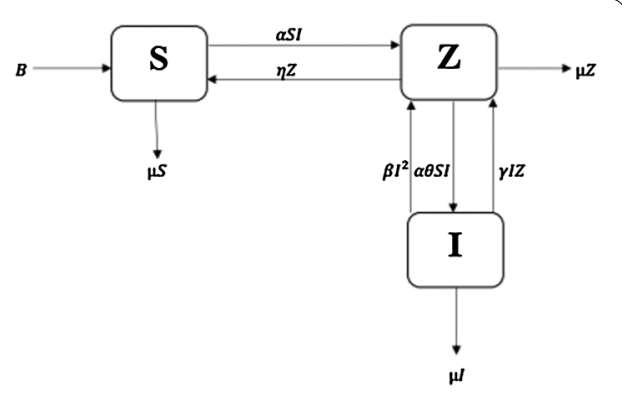

\section{Deterministic meme model}

In this section, we consider the meme transmission model presented in [17]. The variables of meme transmission model at any time $t$ are defined as follows: $S(t)$ : the susceptible class, $I(t)$ : the spreader class, who have tendency to talk about the meme in social interaction, and $Z(t)$ : the stifler class, who have experienced the meme. The flow of the meme model is given in Fig. 1.

The parameters of meme transmission model are defined as follows: $B$ represents the summation of birth and immigration rate of population, $\mu$ represents the rate of emigrant, $\alpha$ is the rate at which the susceptible change their meme class, $\beta$ is the rate at which spreaders become stiflers, $\gamma$ is the rate at which spreaders become stiflers by contacting stiflers, $\theta$ is the rate at which the susceptible become spreaders, $1-\theta$ is the fraction of the susceptible who become stiflers, $\eta$ is the rate at which stiflers become the susceptible again.

The system of ODEs of meme model is as follows:

$$
\begin{aligned}
& \frac{d S}{d t}=B+\eta Z-\alpha S I-\mu S, \\
& \frac{d I}{d t}=\alpha \theta S I-\beta I^{2}-\gamma I Z-\mu I, \\
& \frac{d Z}{d t}=\alpha(1-\theta) S I+\beta I^{2}+\gamma I Z-\eta Z-\mu Z,
\end{aligned}
$$

where $N=S+I+Z$. From system (1)-(3) we have that $N^{\prime}(t)+\mu N(t)=B$, which has the solution $N(t)=e^{-\mu t}+\frac{B}{\mu}$. Therefore, $\lim _{t \rightarrow \infty} N(t)=\frac{B}{\mu}$. Thus, the section for system (1)(3) is $\Gamma=\left\{(S, I, Z): S+I+Z \leq \frac{B}{\mu}, S \geq 0, I \geq 0, Z \geq 0\right\}$. Hence, $\Gamma$ is positively translation invariant.

\subsection{Equilibria of the meme model}

There are two equilibria of meme model (1)-(3) as follows:

$$
\begin{aligned}
& \text { Meme free equilibrium }=(\mathrm{MFE})=\left(\frac{B}{\mu}, 0,0\right), \\
& \text { Meme existence equilibrium }=(\operatorname{MEE})=\left(S_{1}, I_{1}, Z_{1}\right),
\end{aligned}
$$

where

$$
S_{1}=\frac{\beta+\eta Z_{1}}{\alpha I_{1}+\mu}=\frac{1}{\alpha I_{1}}\left[(\mu+\eta) Z_{1}+\mu I_{1}\right], \quad Z_{1}=\frac{-\mu I_{1}}{\left(\alpha I_{1}+\mu\right)+\eta}\left(\frac{\alpha I_{1}}{\mu}+1-\frac{\alpha B}{\mu^{2}}\right),
$$


Table 1 Transition of meme model

\begin{tabular}{ll}
\hline$T_{i}=$ Transitions & $P_{i}=$ Probabilities \\
\hline$T_{1}=[1,0,0]^{\mathrm{T}}$ & $P_{1}=B \Delta t$ \\
$T_{2}=[1,0,-1]^{\mathrm{T}}$ & $P_{2}=\eta Z \Delta t$ \\
$T_{3}=[-1,0,1]^{\mathrm{T}}$ & $P_{3}=\alpha S / \Delta t$ \\
$T_{4}=[-1,0,0]^{\mathrm{T}}$ & $P_{4}=\mu S \Delta t$ \\
$T_{5}=[0,1,-1]^{\mathrm{T}}$ & $P_{5}=\alpha \theta S / \Delta t$ \\
$T_{6}=[0,-1,1]^{\mathrm{T}}$ & $P_{6}=\beta I^{2} \Delta t$ \\
$T_{7}=[0,-1,1]^{\mathrm{T}}$ & $P_{7}=\gamma / Z \Delta t$ \\
$T_{8}=[0,-1,0]^{\mathrm{T}}$ & $P_{8}=\mu / \Delta t$ \\
$T_{9}=[0,0,-1]^{\mathrm{T}}$ & $P_{9}=\mu Z \Delta t$ \\
\hline
\end{tabular}

$$
I_{1}=\frac{\alpha \theta S_{1}-\gamma Z_{1}-\mu}{\beta} .
$$

Note that $R_{\mathrm{o}}^{d}=\frac{\alpha \theta \beta}{\mu^{2}}$ is a deterministic meme threshold number.

\section{Stochastic meme model}

We calculate the transmission probabilities of the model as presented in Table 1.

The expectations of meme model are as follows:

$$
\begin{aligned}
& \mathrm{E}^{*}[\Delta M]=\sum_{i=1}^{9} P_{i} T_{i}, \\
& \text { Expectation }=\mathrm{E}^{*}[\Delta M]=\left[\begin{array}{c}
B+\eta Z-\alpha S I-\mu S \\
\alpha \theta S I-\beta I^{2}-\gamma I Z-\mu I \\
\alpha(1-\theta) S I+\beta I^{2}+\gamma I Z-\eta Z-\mu Z
\end{array}\right] \Delta t, \\
& \text { Variance }=\mathrm{E}^{*}\left[\Delta M \Delta M^{\mathrm{T}}\right]=\sum_{i=1}^{9} P_{i}\left[T_{i}\right]\left[T_{i}\right]^{\mathrm{T}}, \\
& \mathrm{E}^{*}\left[\begin{array}{cc}
\left.\Delta M \Delta M^{\mathrm{T}}\right] \\
0
\end{array}\right. \\
& \left.\quad \begin{array}{ccc}
P_{1}+P_{2}+P_{3}+P_{4} & -P_{2}-P_{3} \\
0 & P_{5}+P_{6}+P_{7}+P_{8} & -P_{5}-P_{6}-P_{7} \\
-P_{2}-P_{3} & -P_{5}-P_{6}-P_{7} & P_{2}+P_{3}+P_{5}+P_{6}+P_{7}+P_{9}
\end{array}\right] \Delta t .
\end{aligned}
$$

So, drift $=G_{1}(M(t), t)=\frac{E^{*}[\Delta M]}{\Delta t}$ and diffusion $=G_{2}(M(t), t)=\sqrt{\frac{E^{*}\left[\Delta M \Delta M^{\mathrm{T}}\right]}{\Delta t}}$ of the model are as follows:

$$
d M(t)=G_{1}(M(t), t) d t+G_{2}(M(t), t) d B(t)
$$

Thus, equation (4) is called stochastic differential equation of the model with initial condition $M(0)=[0.5,0.3,0.2]^{\mathrm{T}}, 0 \leq t \leq T$. Brownian motion is represented by $B(t)$.

\subsection{Euler-Maruyama approach}

The given approach for system (1)-(3) is presented in $[18,19]$ and we shall use Table 2 of parameter values as follows:

$$
M_{n+1}=M_{n}+G_{1}\left(M_{n}, t\right) \Delta t+G_{2}\left(M_{n}, t\right) \Delta B_{n},
$$


Table 2 Parameter values

\begin{tabular}{lll}
\hline Parameters & Values (years) & Source \\
\hline$B$ & 0.05 & {$[17]$} \\
$\mu$ & 0.34 & {$[17]$} \\
$\theta$ & 0.333 & {$[17]$} \\
$\beta$ & 0.05 & {$[17]$} \\
$\alpha$ & $\mathrm{MFE}=1.0125$ & {$[17]$} \\
& $\mathrm{MPE}=10.0125$ & \\
$\eta$ & 0.023 & {$[17]$} \\
$\sigma$ & 0.09 & {$[17]$} \\
\hline
\end{tabular}

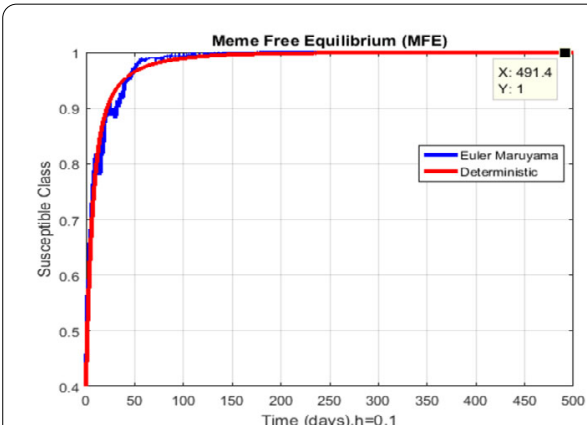

(a)

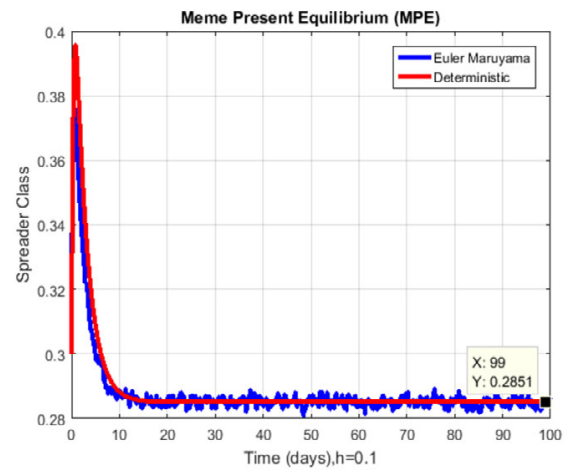

(c)

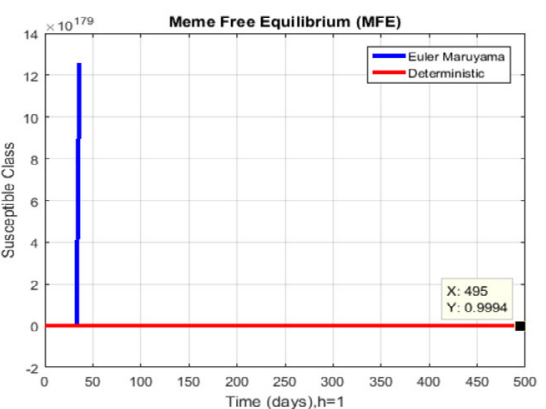

(b)

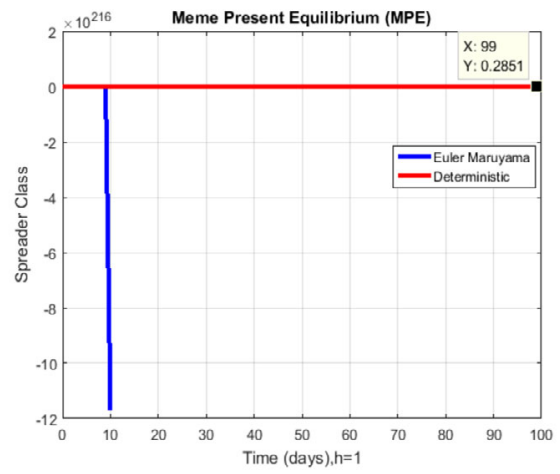

(d)

Figure 2 (a) Susceptible class for MFE at $h=0.1$. (b) Susceptible class for MFE at $h=1$. (c) Spreader class for MPE at $h=0.1$. (d) Spreader class for MPE at $h=1$

where the time step is represented by ' $\Delta t$ ' and $\Delta B_{n}$ is a standard normal distribution, i.e., $\Delta B_{n} \sim \mathrm{N}(0,1)$.

The solution of system (1)-(3), i.e., MFE is $D=\left(\frac{B}{\mu}, 0,0\right)$ and MPE is $E=(0.1107,0.2851$, 0.5991), as presented in Fig. 2.

\section{Parametric noise in the meme model}

In this approach, we select a parameter with small noise as $\alpha d t=\alpha d t+\sigma d B$. So, meme system (1)-(3) is as follows [20]:

$$
\begin{aligned}
& d S=(B+\eta Z-\alpha S I-\mu S) d t-\sigma S I d B, \\
& d I=\left(\alpha \theta S I-\beta I^{2}-\gamma I Z-\mu I\right) d t+\sigma \theta S I d B, \\
& d Z=\left[\left(\alpha(1-\theta) S I+\beta I^{2}+\gamma I Z-\eta Z-\mu Z\right) d t+\sigma(1-\theta) S I d B\right],
\end{aligned}
$$


where $B$ represents Brownian motion and $\sigma$ is the randomness of system (6)-(8). System (6)-(8) is nonintegrable because of Brownian motion. In the coming section, we use different stochastic numerical approaches for system (6)-(8).

\subsection{Stochastic threshold dynamics}

For system (6)-(8) the infected individuals $I(t)$ are said to be extinct if $\lim _{t \rightarrow \infty} I(t)=0$ almost surely.

Let us introduce $R_{\mathrm{o}}^{S}=R_{\mathrm{o}}^{d}-\frac{\sigma^{2} \theta^{2} B^{2}}{2 \mu^{2}}$.

Theorem 4.1 If $\sigma^{2}<\frac{\alpha \mu}{B}$ and $R_{\mathrm{o}}^{S}<1$, then the infected individuals of system (6)-(8) tend to zero exponentially almost surely.

Proof Assume that $(S(t), I(t), Z(t))$ is a solution of system (6)-(8) satisfying the initial value $(S(0), I(0), Z(0)) \in \mathrm{R}_{+}^{3}$ by Ito's formula $f(I)=\ln (I)$.

$$
\begin{aligned}
& d \ln (I)=f^{\prime}(I) d I+\frac{1}{2} f^{\prime \prime}(I) I^{2}\left(\sigma^{2} \theta^{2} S^{2}\right) d t, \\
& d \ln (I)=\frac{1}{I}\left[\left(\alpha \theta S I-\beta I^{2}-\gamma I Z-\mu I\right) d t+\sigma \theta S I d B\right]+\frac{1}{2}\left(\frac{1}{I^{2}}\right) I^{2}\left(\sigma^{2} \theta^{2} S^{2}\right) d t, \\
& d \ln (I)=[(\alpha \theta S-\beta I-\gamma Z-\mu) d t+\sigma \theta S d B]-\frac{\sigma^{2} \theta^{2} S^{2}}{2} d t, \\
& d \ln (I)=\left[\alpha \theta S-\beta I-\gamma Z-\mu-\frac{\sigma^{2} \theta^{2} S^{2}}{2}\right] d t+\sigma \theta S d B, \\
& \ln I(t)=\ln I(0)+\int_{0}^{t}\left(\alpha \theta S-\beta I-\gamma Z-\mu-\frac{\sigma^{2} \theta^{2} S^{2}}{2}\right) d t+\int_{0}^{t} \sigma \theta S d B .
\end{aligned}
$$

If $\sigma^{2}>\frac{\alpha \mu}{B}$,

$$
\begin{aligned}
& \ln I(t) \leq\left(\frac{\alpha^{2} \theta^{2}}{2 \sigma^{2}}-\mu\right) t+M_{1}(t)+\ln I(0), \\
& \frac{\ln I(t)}{t} \leq\left(\frac{\alpha^{2} \theta^{2}}{2 \sigma^{2}}-\mu\right)+\frac{M_{1}(t)}{t}+\frac{\ln I(0)}{t} .
\end{aligned}
$$

If $\lim _{t \rightarrow \infty} \frac{M_{1}(t)}{t}=0$,

$$
\lim _{t \rightarrow \infty} \operatorname{Sup} \frac{I(t)}{t} \leq-\left(\mu-\frac{\alpha^{2} \theta^{2}}{2 \sigma^{2}}\right)<0 .
$$

When $\sigma^{2}>\frac{\alpha^{2} \theta^{2}}{2 \mu}, \lim _{t \rightarrow \infty} I(t)=0$ almost surely.

$$
\text { If } \sigma^{2}<\frac{\alpha \mu}{B} \text {, then }
$$

$$
\begin{aligned}
& \ln I(t) \leq\left(\frac{\alpha \theta \beta}{\mu}-\mu-\frac{\sigma^{2} \theta^{2} B^{2}}{2 \mu^{2}}-\mu\right) t+M_{1}(t)+\ln I(0), \\
& \frac{\ln I(t)}{t} \leq\left(\frac{\alpha \theta \beta}{\mu}-\mu-\frac{\sigma^{2} \theta^{2} B^{2}}{2 \mu^{2}}-\mu\right) t+\frac{M_{1}(t)}{t}+\frac{\ln I(0)}{t}, \\
& \lim _{t \rightarrow \infty} \frac{\ln I(t)}{t} \leq \mu\left(\frac{\alpha \theta \beta}{\mu^{2}}-\frac{\sigma^{2} \theta^{2} B^{2}}{2 \mu^{3}}-1\right) \leq \mu\left[R_{0}^{S}-1\right],
\end{aligned}
$$


then

$$
\begin{aligned}
& \lim _{t \rightarrow \infty} I(t)=0, \quad \text { almost surely, } \\
& R_{\mathrm{o}}^{S}=R_{\mathrm{o}}^{d}-\frac{\sigma^{2} \theta^{2} B^{2}}{2 \mu^{3}}<1 .
\end{aligned}
$$

Note that $R_{\mathrm{o}}^{S}$ is the stochastic meme generation number. The stochastic meme generation number $R_{\mathrm{o}}^{S}=0.9467<1$ means meme free population. The stochastic meme epidemic generation number $R_{\mathrm{o}}^{S}=9.7615>1$ means that the meme is present in the population.

\subsection{Stochastic Euler approach}

The given approach is constructed for system (6)-(8) as follows [20-22]:

$$
\begin{aligned}
& S^{n+1}=S^{n}+h\left[B+\eta Z^{n}-\alpha S^{n} I^{n}-\mu S^{n}-\sigma S^{n} I^{n} \Delta B_{n}\right], \\
& I^{n+1}=I^{n}+h\left[\alpha \theta S^{n} I^{n}-\beta I^{n} I^{n}-\gamma I^{n}-\mu I^{n}+\sigma \theta S^{n} I^{n} \Delta B_{n}\right], \\
& Z^{n+1}=Z^{n}+h\left[\alpha(1-\theta) S^{n} I^{n}+\beta I^{n} I^{n}+\gamma I^{n} Z^{n}-\eta Z^{n}-\mu Z^{n}+\sigma(1-\theta) S^{n} I^{n} \Delta B_{n}\right] .
\end{aligned}
$$

So, $h$ denotes time step size and thus $\Delta B_{n} \sim \mathrm{N}(0,1)$.

\subsection{Stochastic Runge-Kutta approach}

The given approach is constructed for system (6)-(8) as follows [20-22]:

Stage 1

$$
\begin{aligned}
& K_{1}=h\left[B+\eta Z^{n}-\alpha S^{n} I^{n}-\mu S^{n}-\sigma S^{n} I^{n} \Delta B_{n}\right], \\
& M_{1}=h\left[\alpha \theta S^{n} I^{n}-\beta I^{n} I^{n}-\gamma I^{n}-\mu I^{n}+\sigma \theta S^{n} I^{n} \Delta B_{n}\right], \\
& N_{1}=h\left[\alpha(1-\theta) S^{n} I^{n}+\beta I^{n} I^{n}+\gamma I^{n} Z^{n}-\eta Z^{n}-\mu Z^{n}+\sigma(1-\theta) S^{n} I^{n} \Delta B_{n}\right] .
\end{aligned}
$$

Stage 2

$$
\begin{aligned}
K_{2}= & {\left[B+\eta\left(Z^{n}+\frac{N_{1}}{2}\right)-\alpha\left(S^{n}+\frac{K_{1}}{2}\right)\left(I^{n}+\frac{M_{1}}{2}\right)-\mu\left(S^{n}+\frac{K_{1}}{2}\right)\right.} \\
& \left.-\sigma\left(S^{n}+\frac{K_{1}}{2}\right)\left(I^{n}+\frac{M_{1}}{2}\right) \Delta B_{n}\right], \\
M_{2}= & {\left[\alpha \theta\left(S^{n}+\frac{K_{1}}{2}\right)\left(I^{n}+\frac{M_{1}}{2}\right)-\beta\left(I^{n}+\frac{M_{1}}{2}\right)\left(I^{n}+\frac{M_{1}}{2}\right)-\gamma\left(I^{n}+\frac{M_{1}}{2}\right)\right.} \\
& \left.-\mu\left(I^{n}+\frac{M_{1}}{2}\right)+\sigma \theta\left(S^{n}+\frac{K_{1}}{2}\right)\left(I^{n}+\frac{M_{1}}{2}\right) \Delta B_{n}\right], \\
N_{2}= & h\left[\alpha(1-\theta)\left(S^{n}+\frac{K_{1}}{2}\right)\left(I^{n}+\frac{M_{1}}{2}\right)+\beta\left(I^{n}+\frac{M_{1}}{2}\right)\left(I^{n}+\frac{M_{1}}{2}\right)\right. \\
& +\gamma\left(I^{n}+\frac{M_{1}}{2}\right)\left(Z^{n}+\frac{N_{1}}{2}\right)-(\eta+\mu)\left(Z^{n}+\frac{N_{1}}{2}\right) \\
& \left.+\sigma(1-\theta)\left(S^{n}+\frac{K_{1}}{2}\right)\left(I^{n}+\frac{M_{1}}{2}\right) \Delta B_{n}\right] .
\end{aligned}
$$


Stage 3

$$
\begin{aligned}
K_{3}= & {\left[B+\eta\left(Z^{n}+\frac{N_{2}}{2}\right)-\alpha\left(S^{n}+\frac{K_{2}}{2}\right)\left(I^{n}+\frac{M_{2}}{2}\right)-\mu\left(S^{n}+\frac{K_{2}}{2}\right)\right.} \\
& \left.-\sigma\left(S^{n}+\frac{K_{2}}{2}\right)\left(I^{n}+\frac{M_{2}}{2}\right) \Delta B_{n}\right], \\
M_{3}= & {\left[\alpha \theta\left(S^{n}+\frac{K_{2}}{2}\right)\left(I^{n}+\frac{M_{2}}{2}\right)-\beta\left(I^{n}+\frac{M_{2}}{2}\right)\left(I^{n}+\frac{M_{2}}{2}\right)-\gamma\left(I^{n}+\frac{M_{2}}{2}\right)\right.} \\
& \left.-\mu\left(I^{n}+\frac{M_{2}}{2}\right)+\sigma \theta\left(S^{n}+\frac{K_{2}}{2}\right)\left(I^{n}+\frac{M_{2}}{2}\right) \Delta B_{n}\right], \\
N_{3}= & {\left[\alpha(1-\theta)\left(S^{n}+\frac{K_{2}}{2}\right)\left(I^{n}+\frac{M_{2}}{2}\right)+\beta\left(I^{n}+\frac{M_{2}}{2}\right)\left(I^{n}+\frac{M_{2}}{2}\right)\right.} \\
& +\gamma\left(I^{n}+\frac{M_{2}}{2}\right)\left(Z^{n}+\frac{N_{2}}{2}\right)-(\eta+\mu)\left(Z^{n}+\frac{N_{2}}{2}\right) \\
& \left.+\sigma(1-\theta)\left(S^{n}+\frac{K_{2}}{2}\right)\left(I^{n}+\frac{M_{2}}{2}\right) \Delta B_{n}\right] .
\end{aligned}
$$

Stage 4

$$
\begin{aligned}
K_{4}= & h\left[B+\eta\left(Z^{n}+N_{3}\right)-\alpha\left(S^{n}+K_{3}\right)\left(I^{n}+M_{3}\right)-\mu\left(S^{n}+K_{3}\right)\right. \\
& \left.-\sigma\left(S^{n}+K_{3}\right)\left(I^{n}+M_{3}\right) \Delta B_{n}\right], \\
M_{4}= & h\left[\alpha \theta\left(S^{n}+K_{3}\right)\left(I^{n}+M_{3}\right)-\beta\left(I^{n}+M_{3}\right)\left(I^{n}+M_{3}\right)-\gamma\left(I^{n}+M_{3}\right)\right. \\
& \left.-\mu\left(I^{n}+M_{3}\right)+\sigma \theta\left(S^{n}+K_{3}\right)\left(I^{n}+M_{3}\right) \Delta B_{n}\right], \\
N_{4}= & h\left[\alpha(1-\theta)\left(S^{n}+K_{3}\right)\left(I^{n}+M_{3}\right)+\beta\left(I^{n}+M_{3}\right)\left(I^{n}+M_{3}\right)+\gamma\left(I^{n}+M_{3}\right)\left(Z^{n}+N_{3}\right)\right. \\
& \left.-(\eta+\mu)\left(Z^{n}+N_{3}\right)+\sigma(1-\theta)\left(S^{n}+K_{3}\right)\left(I^{n}+M_{3}\right) \Delta B_{n}\right] .
\end{aligned}
$$

Final stage

$$
\left.\begin{array}{l}
S^{n+1}=S^{n}+\frac{1}{6}\left[K_{1}+2 K_{2}+2 K_{3}+K_{4}\right], \\
I^{n+1}=I^{n}+\frac{1}{6}\left[M_{1}+2 M_{2}+2 M_{3}+M_{4}\right], \\
Z^{n+1}=Z^{n}+\frac{1}{6}\left[N_{1}+2 N_{2}+2 N_{3}+N_{4}\right] .
\end{array}\right\}
$$

So, $h$ represents time step size and thus $\Delta B_{n} \sim \mathrm{N}(0,1)$.

\subsection{Stochastic NSFD approach}

The given approach is constructed for system (6)-(8) as follows [20-22]:

$$
\begin{aligned}
S^{n+1} & =\frac{S^{n}+h B+h \eta Z^{n}}{1+h \alpha I^{n}+h \mu+h \sigma I^{n} \Delta B_{n}}, \\
I^{n+1} & =\frac{I^{n}+h \alpha \theta S^{n} I^{n}+h \sigma \theta S^{n} I^{n} \Delta B_{n}}{1+h \beta I^{n}+h \gamma+h \mu},
\end{aligned}
$$




$$
Z^{n+1}=\frac{Z^{n}+h \alpha(1-\theta) S^{n} I^{n}+h \beta I^{n} I^{n}+h \gamma I^{n} Z^{n}+h \sigma(1-\theta) S^{n} I^{n} \Delta B_{n}}{1+h \eta+h \mu} .
$$

So, $h$ represents time step size and thus $\Delta B_{n} \sim \mathrm{N}(0,1)$.

4.4.1 Convergence analysis

We give the following theorems in support of the given analysis.

Theorem 4.2 The given initial value system $\left(S^{n}(0), I^{n}(0), Z^{n}(0)\right) \in \mathrm{R}_{+}^{3}$, so system $(14)-(16)$ has a unique nonnegative solution $\left(S^{n}, I^{n}, Z^{n}\right) \in \mathrm{R}_{+}^{3}$ on $n \geq 0$, almost surely.

Theorem 4.3 The region

$$
\Omega=\left\{\left(S^{n}, I^{n}, Z^{n}\right) \in \mathrm{R}_{+}^{3}: S^{n} \geq 0, I^{n} \geq 0, Z^{n} \geq 0, S^{n}+I^{n}+Z^{n} \leq \frac{B}{\mu}\right\} \text { for all } n \geq 0
$$

is a nonnegative invariant set for system (14)-(16).

Proof System (14)-(16) may be written as follows:

$$
\begin{aligned}
& \frac{S^{n+1}-S^{n}}{h}=\left[B+\eta Z^{n}-\alpha S^{n} I^{n}-\mu S^{n}-\sigma S^{n} I^{n} \Delta B_{n}\right], \\
& \frac{I^{n+1}-I^{n}}{h}=\left[\alpha \theta S^{n} I^{n}-\beta I^{n} I^{n}-\gamma I^{n}-\mu I^{n}+\sigma \theta S^{n} I^{n} \Delta B_{n}\right], \\
& \frac{Z^{n+1}-Z^{n}}{h}=\left[\alpha(1-\theta) S^{n} I^{n}+\beta I^{n} I^{n}+\gamma I^{n} Z^{n}-\eta Z^{n}-\mu Z^{n}+\sigma(1-\theta) S^{n} I^{n} \Delta B_{n}\right], \\
& \frac{\left(S^{n+1}+I^{n+1}+Z^{n+1}\right)-\left(S^{n}+I^{n}+Z^{n}\right)}{h}=B-\mu\left(S^{n}+I^{n}+Z^{n}\right), \\
& \left(S^{n+1}+I^{n+1}+Z^{n+1}\right)-\left(S^{n}+I^{n}+Z^{n}\right)=h B-h \mu\left(S^{n}+I^{n}+Z^{n}\right), \\
& \left(S^{n+1}+I^{n+1}+Z^{n+1}\right) \leq \frac{B}{\mu},
\end{aligned}
$$

almost surely.

Theorem 4.4 The discretized system (14)-(16) has the same equilibria as the continuous system (6)-(8) for all $n \geq 0$.

Proof For solving system (14)-(16), we get two states as follows:

$$
\begin{aligned}
& \text { MFE i.e. } D_{3}=\left(S^{n}, I^{n}, Z^{n}\right)=\left(\frac{B}{\mu}, 0,0\right), \\
& \text { MPE i.e. } E_{3}=\left(S^{n}, I^{n}, Z^{n}\right)
\end{aligned}
$$

where

$$
\begin{aligned}
& S^{n}=\frac{\beta+\eta Z^{n}}{\alpha I^{n}+\mu}=\frac{1}{\alpha I^{n}}\left[(\mu+\eta) Z^{n}+\mu I^{n}\right], \\
& Z^{n}=\frac{-\mu I^{n}}{\left(\alpha I^{n}+\mu\right)+\eta}\left(\frac{\alpha I^{n}}{\mu}+1-\frac{\alpha B}{\mu^{2}}\right),
\end{aligned}
$$




$$
I^{n}=\frac{\alpha \theta S^{n}-\gamma Z^{n}-\mu}{\beta}
$$

almost surely.

Theorem 4.5 The eigenvalues of the discretized system (14)-(16) lie in the unit circle for all $n \geq 0$.

Proof Let us suppose $F, G$, and $H$ from system (14)-(16) as follows:

$$
\begin{aligned}
& F=\frac{S+h B+h \eta Z}{1+h \alpha+h \mu+h \sigma I \Delta B_{n}}, \\
& G=\frac{I+h \alpha \theta S I+h \sigma \theta S I \Delta B_{n}}{1+h \beta I+h(\gamma+\mu)}, \\
& H=\frac{Z+h \alpha(1-\theta) S I+h \beta I^{2}+h \gamma I Z+h \sigma(1-\theta) S I \Delta B_{n}}{1+h(\eta+\mu)}, \\
& \frac{\partial F}{\partial S}=\frac{1}{1+h \alpha+h \mu+h \sigma I \Delta B_{n}}, \quad \frac{\partial F}{\partial I}=\frac{-h \sigma \Delta B_{n}(S+h B+h \eta Z)}{\left[1+h \alpha+h \mu+h \sigma I \Delta B_{n}\right]^{2}}, \\
& \frac{\partial F}{\partial Z}=\frac{h \eta}{1+h \alpha+h \mu+h \gamma I \Delta B_{n}}, \quad \frac{\partial G}{\partial S}=\frac{h \alpha \theta I+h \sigma \theta I \Delta B_{n}}{1+h \beta I+h(\gamma+\mu)}, \\
& \frac{\partial G}{\partial I}=\frac{[1+h \beta I+h(\gamma+\mu)]\left[1+h \alpha \theta S+h \sigma \theta S \Delta B_{n}\right]-h \beta\left[I+h \alpha \theta S I+h \sigma \theta S I \Delta B_{n}\right]}{\left[1+h \alpha+h \mu+h \sigma I \Delta B_{n}\right]^{2}}, \\
& \frac{\partial G}{\partial Z}=0, \quad \frac{\partial H}{\partial S}=\frac{h \alpha(1-\theta) S I+h \sigma(1-\theta) I \Delta B_{n}}{1+h(\eta+\mu)}, \\
& \frac{\partial H}{\partial I}=\frac{h \alpha(1-\theta) S+2 h \beta I+h \gamma Z+h \sigma(1-\theta) S \Delta B_{n}}{1+h(\eta+\mu)}, \quad \frac{\partial H}{\partial Z}=\frac{1+h \gamma I}{1+h(\eta+\mu)} .
\end{aligned}
$$

The given Jacobian matrix at $D_{1}=(S, I, Z)=\left(\frac{B}{\mu}, 0,0\right)$ and $R_{\mathrm{o}}^{S}<1$ is as follows:

$$
\begin{aligned}
& J=\left[\begin{array}{lll}
\frac{\partial F}{\partial S} & \frac{\partial F}{\partial I} & \frac{\partial F}{\partial Z} \\
\frac{\partial G}{\partial S} & \frac{\partial G}{\partial I} & \frac{\partial G}{\partial Z} \\
\frac{\partial H}{\partial S} & \frac{\partial H}{\partial I} & \frac{\partial H}{\partial Z}
\end{array}\right], \\
& J\left(\frac{B}{\mu}, 0,0\right)=\left[\begin{array}{ccc}
\frac{1}{1+h \alpha+h \mu} & \frac{-h \sigma \Delta B_{n}\left(\frac{B}{\mu}+h B\right)}{\left[1+h \alpha+h \mu+h \sigma I \Delta B_{n}\right]^{2}} & \frac{h \eta}{1+h \alpha+h \mu} \\
0 & \frac{1+\frac{h \theta B}{\mu}+\frac{h \theta \theta B \Delta B_{n}}{1+h(\eta+\mu)}}{1+h(1+\mu)} & 0 \\
0 & \frac{h \alpha(1-\theta) B+h \sigma(1-\theta) B \Delta B_{n}}{1+h(\eta)} & \frac{1}{1+h(\eta+\mu)}
\end{array}\right] .
\end{aligned}
$$

The eigen values are

$$
\begin{aligned}
& \lambda_{1}=\frac{1}{1+h \alpha+h \mu}<1, \quad \lambda_{2}=\frac{\mu+h \alpha \theta B+h \sigma \theta B \Delta B_{n}}{\mu[1+h \alpha+h \mu]}<1 \quad \text { when } R_{\mathrm{o}}^{S}<1 ; \\
& \lambda_{3}=\frac{1}{1+h(\alpha+\mu)}<1 .
\end{aligned}
$$

So, all the eigenvalues of a given matrix are in the range of unit circle. So, system (14)-(16) is linearizable around $D_{1}$. 


\subsection{Contrast section}

We present a contrast of the aforesaid stochastic approaches in what follows.

\subsection{Covariance of meme model}

In this section, we discuss the covariance of meme transmission epidemic model among its compartments. For this, we calculate the correlation coefficients and outcomes as described in Table 3. The solutions in Table 3 show an inverse relationship among the susceptible class and others. The given model will attain meme free equilibria if the susceptible class has inverse relation, which means that the decrease in this compartment will eventually lead to the increase in other compartments.

\section{Results and discussion}

For discretization parameter $h=0.1$, we see that Euler-Maruyama (EM) approach meets the equilibria of the meme model; on the other hand, the ODEs solution is the mean of its solution; this can be seen in Fig. 2(a) and Fig. 2(c). The Euler-Maryuama (EM) approach fails to sustain positivity and shows divergence when we increase the step size for both points; this can be seen in Fig. 2(b) and Fig. 2(d). For discretization parameter $h=0.1$, we

Table 3 Correlation coefficients

\begin{tabular}{lll}
\hline Sub-populations & Correlation coefficient $(\rho)$ & Relationship \\
\hline$(S, I)$ & -0.0255 & Inverse \\
$(I, Z)$ & -0.6288 & Inverse \\
$(S, Z)$ & -0.7604 & Inverse \\
\hline
\end{tabular}

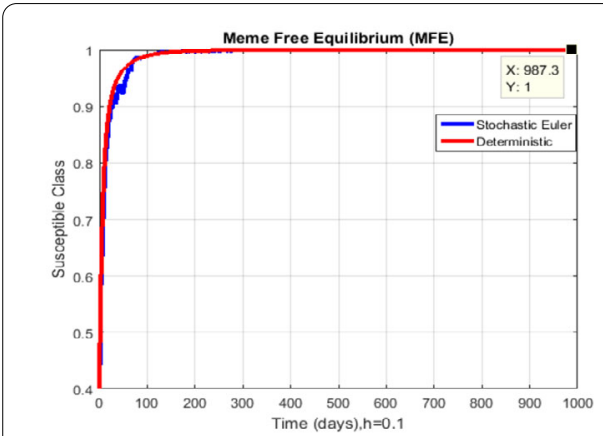

(a)

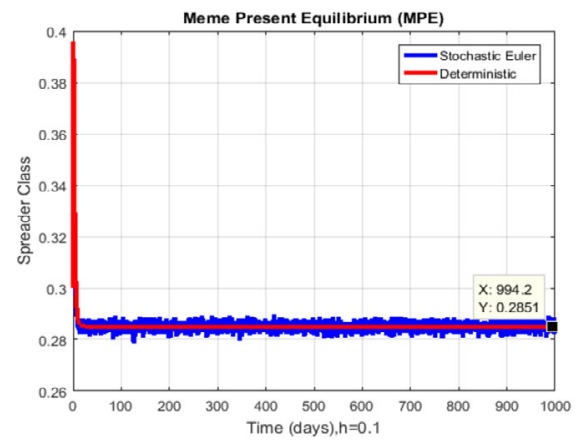

(c)

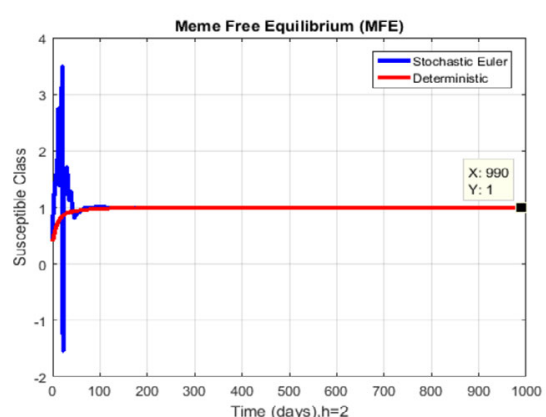

(b)

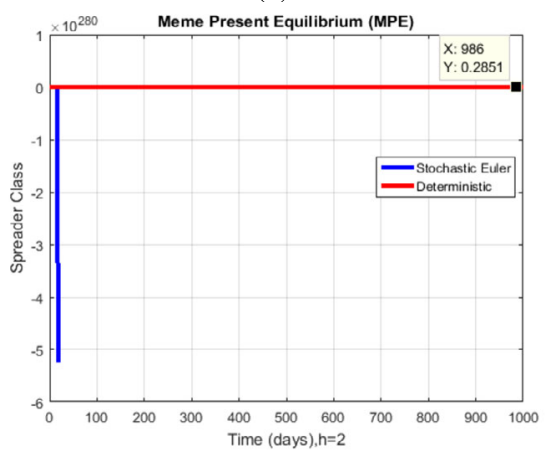

(d)

Figure 3 (a) Susceptible class for MFE at $h=0.1$. (b) Susceptible class for MFE at $h=2$. (c) Spreader class for MPE at $h=0.1$. (d) Spreader class for MPE at $h=2$ 


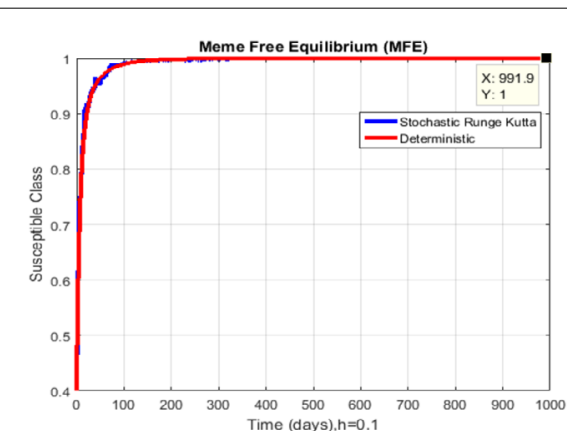

(a)



(c)

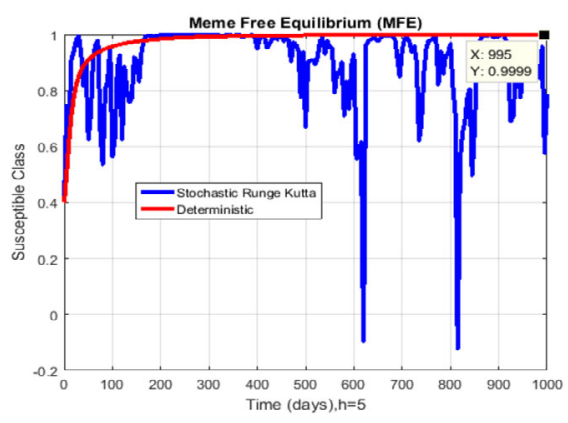

(b)

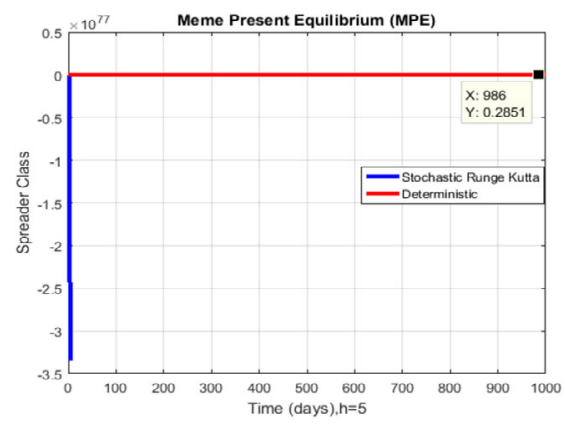

(d)

Figure 4 (a) Susceptible class for MFE at $h=0.1$. (b) Susceptible class for MFE at $h=5$. (c) Spreader class for MPE at $h=0.1$. (d) Spreader class for MPE at $h=5$

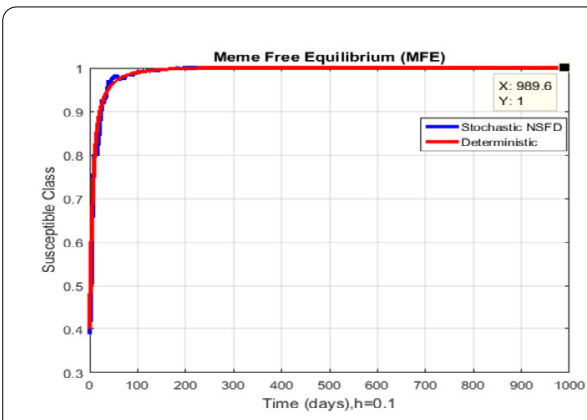

(a)

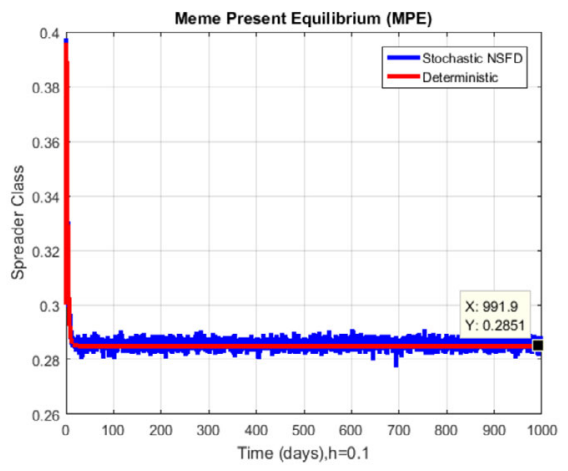

(c)

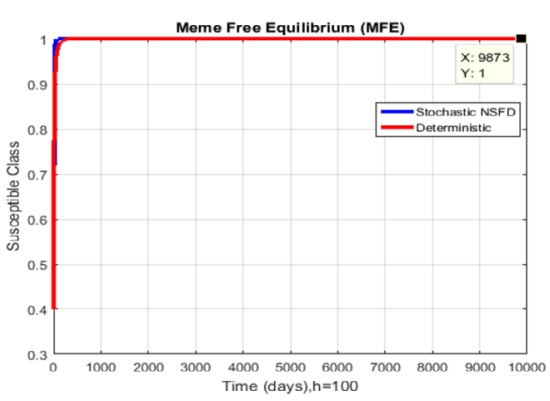

(b)

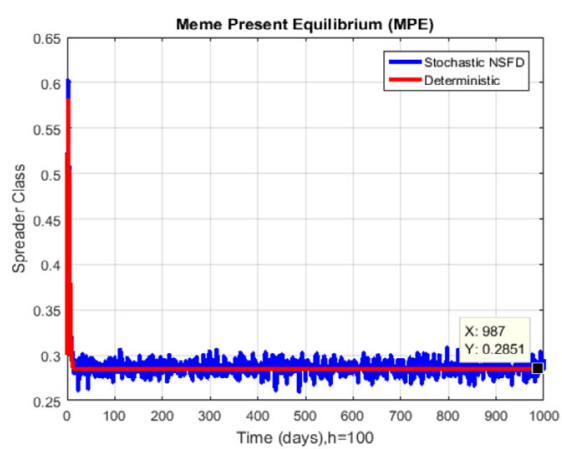

(d)

Figure 5 (a) Susceptible class for MFE at $h=0.1$. (b) Susceptible class for MFE at $h=100$. (c) Spreader class for MPE at $h=0.1$. (d) Spreader class for MPE at $h=100$ 


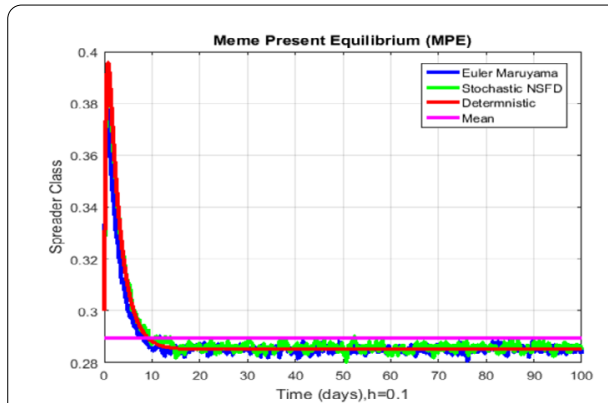

(a)

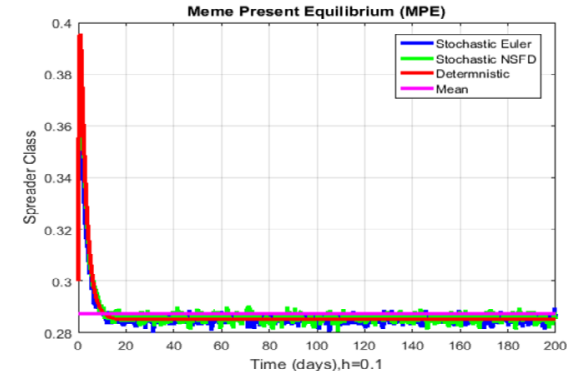

(c)

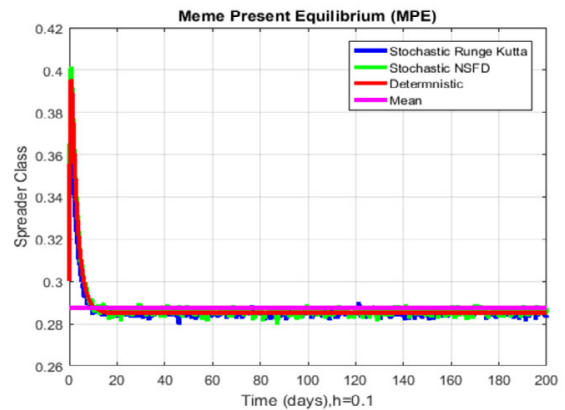

(e )

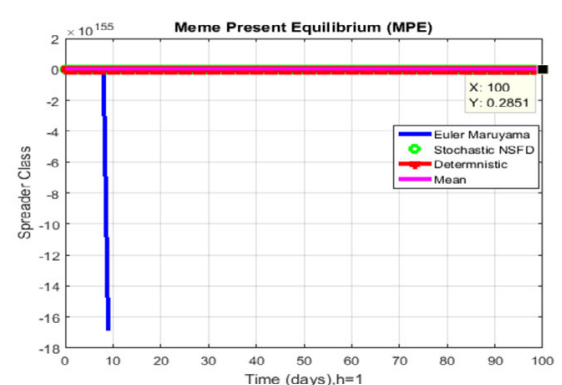

(b)

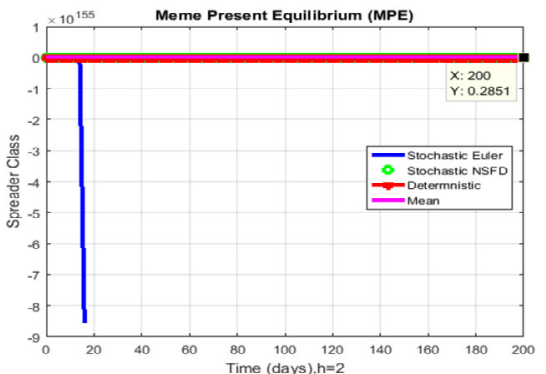

(d)

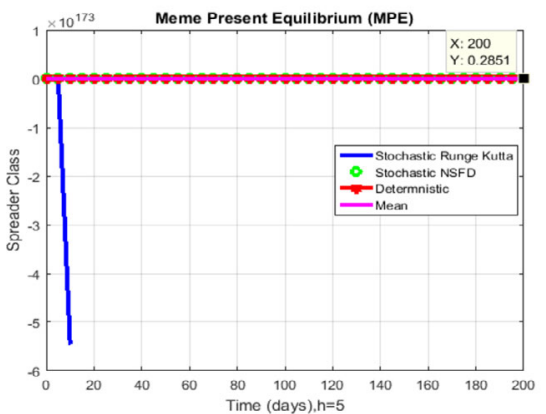

(f)

Figure 6 (a) Spreader class at $h=0.1$ with Euler-Maruyama. (b) Spreader class at $h=1$ with Euler-Maruyama. (c) Spreader class at $h=0.1$ with stochastic Euler. (d) Spreader class at $h=2$ with stochastic Euler. (e) Spreader class at $h=0.1$ with stochastic Runge-Kutta. (f) Spreader class at $h=5$ with stochastic Runge-Kutta

see the stochastic Euler approach convergence to the equilibrium point, this can be seen in Fig. 3(a) and Fig. 3(c). For both meme free and meme present equilibria, the stochastic Euler approach fails to preserve the structure of the model when we increase time step size, this can be seen in Fig. 3(b) and Fig. 3(d). For step size $h=0.1$, we see that the stochastic Runge-Kutta approach converges to meme free equilibrium and meme-present equilibrium, this can be seen graphically in Fig. 4(a) and Fig. 4(c). The stochastic Runge-Kutta approach fails to maintain stability and positivity for both meme free and meme present equilibrium points when we increase the step size; this change happens in Fig. 4(b) and Fig. 4(d).

Thus the aforementioned stochastic approaches do not retain all the dynamical properties $[15,16,18]$. For discretization parameter $h=0.1$ and $h=100$, the stochastic NSFD approach converges for both equilibrium points for any parameter; this can be seen in Fig. 5. The contrast of numerical approaches presented in Fig. 6 proved the efficiency of the proposed stochastic nonstandard finite difference approach. 


\section{Conclusion and directions}

After this analysis, we can give the idea that SDEs analysis of a meme model is suitable as compared to ODEs analysis. All stochastic approaches violate dynamical properties. These approaches give nonpositive and unbounded solutions. This type of dynamics has no significance in physical systems. We have introduced stochastic NSFD approach for this type of modeling under the assumption discussed by Mickens in the stochastic context [14-16]. This approach always satisfies the dynamical properties such as dynamical consistency, nonnegativity, and boundedness of a model. In the future, we shall extend this idea in the field of all types of social systems. Also, we shall construct fractional order social interaction models [23].

\section{Acknowledgements}

We are thankful for the appreciated remarks from anonymous referees.

\section{Funding}

We have no funding for this article.

\section{Abbreviations}

MFE, Meme Free Equilibrium; MPE, Meme Present Equilibrium; ODEs, Ordinary Differential Equations; SDE, Stochastic Differential Equation; NSFD, Nonstandard Finite Difference.

Availability of data and materials

The data and resources files are accessible.

Competing interests

There are no competing interests against this article.

Authors' contributions

We have equal contribution, and careful reading has been done by all the authors. All authors read and approved the final manuscript.

\section{Author details}

${ }^{1}$ Stochastic Analysis and Optimization Research Group, Department of Mathematics, Air University, PAF Complex E-9, Islamabad, Pakistan. ${ }^{2}$ Faculty of Engineering, University of Central Punjab, Lahore, Pakistan. ${ }^{3}$ Department of Mathematics, Cankaya University, Ankara, Turkey. ${ }^{4}$ Department of Medical Research, China Medical University Hospital, China Medical University, Taichung, Taiwan. ${ }^{5}$ Institute of Space Sciences, Magurele-Bucharest, Romania.

\section{Publisher's Note}

Springer Nature remains neutral with regard to jurisdictional claims in published maps and institutional affiliations.

Received: 19 November 2019 Accepted: 17 March 2020 Published online: 25 April 2020

\section{References}

1. Dawkins, R.: The Selfish Gene, 2nd edn. Oxford University Press, London (1989)

2. Dietz, K.: Epidemics and rumors a survey. J. R. Stat. Soc. A 130(4), 505-528 (1967)

3. Cane, V.: A note on the size of epidemics and the number of people hearing a rumor. J. R. Stat. Soc. B 28(3), 487-490 (1966)

4. Kawachi, K., Seki, M., Yoshida, H., Otake, Y., Warashina, K., Ueda, H.: A rumor transmission model with various contact interactions. J. Theor. Biol. 253(1), 55-60 (2008)

5. Bettencourt, L., Cintron, A.A., Kaiser, D., Chavez, C.C.: The power of a good idea: quantitative modeling of the spread of ideas from epidemiological models. Physica A 364, 513-536 (2006)

6. Wang, L., Wood, B.: An epidemiological approach to model the viral propagation of memes. Appl. Math. Model. 35(11), 5442-5447 (2011)

7. Piqueira, J.: Rumor propagation model an equilibrium study. Math. Probl. Eng. 2010, Article ID 631357 (2010). https://doi.org/10.1155/2010/631357

8. Huang, W.: On rumor spreading with skepticism and denial. Working paper (2011)

9. Zhao, L., Wang, Q., Cheng, J., Chen, Y., Wang, J., Huang, W.: Rumor spreading model with consideration of forgetting mechanism a case of online blogging LiveJournal. Physica A 390(13), 2619-2625 (2011)

10. Zhao, L., Wang, X., Qiu, X., Wang, J.: A model for the spread of rumors in Barrat-Barthelemy-Vespignani (BBV) networks. Physica A 392(21), 5542-5551 (2013)

11. Huo, L., Huang, P., Guo, C.: Analyzing the dynamics of a rumor transmission model with incubation. Discrete Dyn. Nat. Soc. 2012, Article ID 328151 (2012). https://doi.org/10.1155/2012/328151

12. Thompson, K., Estrada, R., Daugherty, D., Cintron, A.A.: A deterministic approach to the spread of rumors. Working paper (2003) 
13. Amoudi, R., Sheikh, S., Tuwairqi, S.: Qualitative behavior of solutions to a mathematical model of memes transmission. Int. J. Appl. Math. Res. 3(1), 36-44 (2014)

14. Mickens, R.E.: A fundamental principle for constructing nonstandard finite difference schemes for differential equations. J. Differ. Equ. Appl. 11(7), 645-653 (2005)

15. Mickens, R.E.: Nonstandard Finite Difference Models of Differential Equations. World Scientific, Singapore (1994)

16. Mickens, R.E.: Advances in Applications of Nonstandard Finite Difference Schemes. World Scientific, Singapore (1992)

17. Amoudi, R., Tuwairqi, S., Sheikh, S.: Stability analysis of a variable meme transmission model. Int. J. Adv. Math. Sci. 2, 107-115 (2014)

18. Maruyama, G.: Continuous Markov processes and stochastic equations. Rend. Circ. Mat. Palermo 4(1), 48-90 (1955)

19. Higham, D.J.: An algorithmic introduction to numerical simulation of stochastic differential equations. SIAM Rev. 43(3), 525-546 (2001)

20. Arif, M.S., Raza, A., Rafiq, M., Bibi, M.: A reliable numerical analysis for stochastic hepatitis B virus epidemic model with the migration effect. Iran. J. Sci. Technol., Trans. A, Sci. 43(5), 2477-2492 (2019)

21. Raza, A., Arif, M.S., Rafiq, M.: A reliable numerical analysis for stochastic dengue epidemic model with incubation period of virus. Adv. Differ. Equ. 2019(1), 32 (2019)

22. Arif, M.S., Raza, A., Rafiq, M., Bibi, M., Fayyaz, R., Naz, M., Javed, U.: A reliable stochastic numerical analysis for typhoid fever incorporating with protection against infection. Comput. Mater. Continua 59(3), 787-804 (2019)

23. Singh, J., Kumar, D., Baleanu, D.: New aspects of fractional Biswas-Milovic model with Mittag-Leffler law. Math. Model Nat. Phenom. 14(3), 303 (2019)

\section{Submit your manuscript to a SpringerOpen ${ }^{\circ}$ journal and benefit from:}

- Convenient online submission

- Rigorous peer review

- Open access: articles freely available online

- High visibility within the field

- Retaining the copyright to your article

Submit your next manuscript at $\boldsymbol{~ s p r i n g e r o p e n . c o m ~}$ 